\title{
Be mindful of the central nervous system in Burkitt lymphoma
}

\section{Mark Roschewski}

Lymphoid Malignancies Branch, Center for Cancer Research, National Cancer Institute, Bethesda, MD, USA

E-mail: MARK ROSCHEWSKI - mark.roschewski@nih.gov

doi:10.3324/haematol.2020.278181

I $\mathrm{n}$ this issue of Haematologica, Zayac et al. illustrate the adverse prognostic impact of central nervous system (CNS) involvement for adult patients with Burkitt lymphoma (BL). ${ }^{1}$ The authors reported data from a retrospective series of 641 patients treated at 30 institutions in the USA and identified 120 (19\%) patients with baseline CNS involvement. In the majority of cases (81\%), the leptomeninges were involved, $17 \%$ had brain parenchymal lesions, and the site was unspecified in $2 \%$. The risk was not uniformly distributed as patients with concomitant human immunodeficiency virus (HIV) infection had CNS involvement in $30 \%$ of cases whereas extranodal disease (particularly testicular involvement), poor performance status, and markedly elevated lactate dehydrogenase level were strongly correlated with CNS spread in HIV-negative patients. Most patients $(89 \%)$ were treated with one of three standard frontline BL regimens: CODOX-M/IVAC, HyperCVAD/MA, and DA-EPOCH-R, presumably all with curative intent. ${ }^{2.4}$ Notably, active CNS involvement was evenly distributed across the three regimens, although this was not the case for other risk factors. The data clearly demonstrated that baseline CNS involvement

identified patients who are difficult to cure with standard frontline approaches as they had lower rates of complete response $(59 \%$ vs. $77 \% ; P<0.001)$ and a significantly lower 3 -year overall survival $(49 \%$ vs. $74 \%$; $P<0.001)$ compared to patients without baseline CNS involvement. Importantly, the negative prognostic impact of baseline CNS involvement was significant for all three regimens.

A second focus of the study was predictors of CNS recurrence in 570 patients treated with the three standard regimens. The overall rate of disease recurrence was $26 \%$ and the recurrence involved the CNS in $23 \%$ of cases. Most of these recurrences ( $82 \%$ ) were isolated to the CNS without concomitant systemic recurrence and $87 \%$ occurred within the first year of treatment. These data strongly suggest that current strategies typically fail to eradicate active baseline CNS disease rather than the CNS being a sanctuary site for late relapses. Indeed, patients with baseline CNS involvement had the highest CNS recurrence rate of $18 \%$. Considered by regimen, patients who received DA-EPOCH-R had a $35 \%$ rate of CNS recurrence among those with baseline CNS disease. These data illustrate that baseline CNS involvement is a

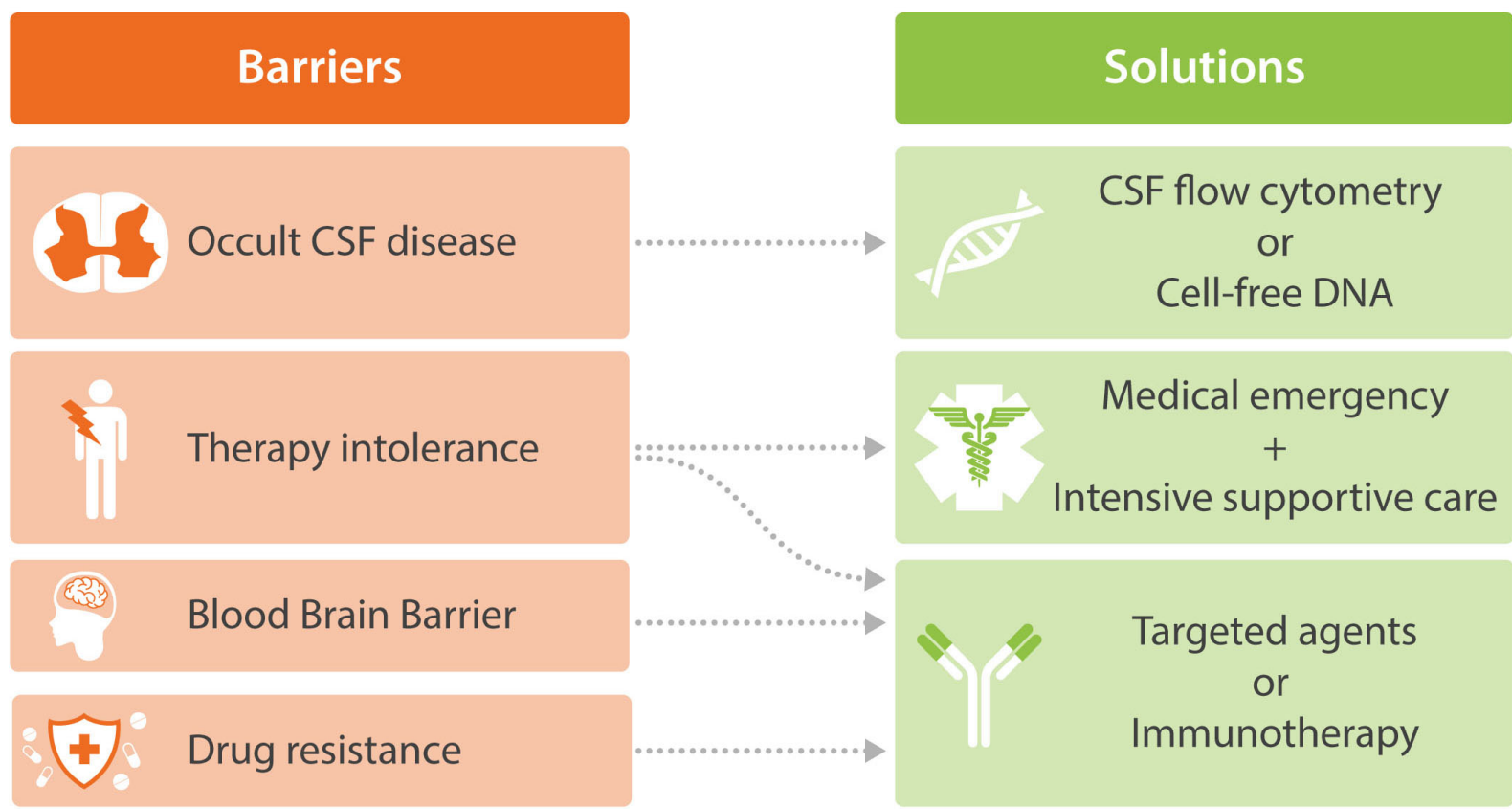

Figure 1. Multiple barriers prevent current strategies from effectively treating and preventing central nervous system disease in adults with Burkitt lymphoma. Routine cytology is insufficiently sensitive for the detection of occult lymphoma cells in the cerebrospinal fluid (CSF) and widespread adoption of more sensitive techniques such as CSF flow cytometry or the development of assays that detect cell-free tumor DNA in the CSF are potential solutions. Therapy intolerance is a barrier unique to adults since pediatric regimens that employ high intensity chemotherapy and are highly effective in children and young adults with central nervous system (CNS) disease are often not tolerated by older patients or those with co-morbid conditions. Treating "possible Burkitt lymphoma (BL)" as a medical emergency with rapid employment of aggressive supportive care can improve performance status to allow for proper CNS-directed interventions and reduce the risk of early toxic death. The blood-brain barrier limits the use of many chemotherapy agents, but novel pathway inhibitors and immunotherapy agents with activity in BL are in clinical development, are often more tolerable, effectively cross the blood-brain barrier, and may overcome chemotherapy resistance. A more complete understanding of the genetic basis for mechanisms of drug resistance in BL may allow the development of additional novel agents that penetrate the CNS. 
greater problem in real-world practice than observed in clinical trials, and that isolated CNS recurrence is a common cause of treatment failure in adults with BL. The contributing factors are multiple and one should not conclude that the problem is readily addressed by choice of frontline regimen. In reality, the absence of highly effective CNS-penetrating agents is a major barrier, and new strategies should focus on this limitation (Figure 1). In the meantime, optimal management mandates being ever mindful of CNS detection, prophylaxis, and treatment.

The first important question is whether high-dose methotrexate-containing regimens such as CODOXM/IVAC or HyperCVAD/MA are superior to DA-EPOCH$\mathrm{R}$ in patients with CNS disease. The inherent selection bias of this retrospective study design precludes a definitive answer since the patients who received DA-EPOCH$\mathrm{R}$ were older and were more likely to have a poor performance status. ${ }^{5}$ It is unclear whether these patients would have even been candidates for highly dose-intensive regimens since toxicities rise sharply with age. ${ }^{6,7}$ In a multicenter study of DA-EPOCH-R in 113 adults with BL, we reported that baseline CNS involvement was associated with an inferior progression-free survival. ${ }^{4}$ Importantly, these patients had equal risk of disease progression as well as early toxic death due to sepsis compared to patients without CNS disease. Intensification of chemotherapy may improve control of CNS disease, but will increase the risk of early toxic death. In reality, only a prospective randomized study, such as the HOVON 127 trial (EudraCT number: 2013-004394-27), can adequately address the question of optimal therapy selection for adults with $\mathrm{BL}$ across various subsets.

Careful review of the data in the report from Zayac et al. and in the published literature suggests that additional barriers contribute to this complex problem (Figure 1). An important component of proper use of DA-EPOCH-R for $\mathrm{BL}$ is a risk-adapted approach that includes a careful analysis of the cerebrospinal fluid (CSF) with flow cytometry to detect occult disease. Using this approach, patients who are positive by flow cytometry are treated with an intensive intrathecal chemotherapy schedule while patients who are negative by flow cytometry receive less intrathecal treatment as CNS prophylaxis. Given the nature of the retrospective study by Zayac et al., the proper use of CSF flow cytometry to identify and adjust treatment with DA-EPOCH-R is unknown as is the use of brain imaging and CSF cytology. The absence of proper CNS evaluation in this series confounds any interpretation that DA-EPOCH-R is less effective than other highdose regimens. Furthermore, only $45 \%$ of the patients received the schedule of intrathecal therapy as published. Lastly, intrathecal chemotherapy alone would not be adequate therapy for brain parenchymal lesions and these patients have been excluded from clinical trials of DAEPOCH-R; yet four patients in the study discussed had brain parenchymal lesions and received DA-EPOCH-R, which contributed to the high rate of CNS recurrence.
The authors adequately address the limitations of their data and should be commended for performing a sobering analysis of outcomes in adults with BL which we commonly consider "highly curable." In reality, these data illustrate that we successfully cure fewer adult BL patients than suggested by clinical trials, although the proper implementation of the treatment regimens in general practice likely contributes to this disparity. Advances in the detection of occult CSF disease, including assays for cellfree tumor DNA, may be important in future studies. Perhaps most importantly, these data highlight that no matter which chemotherapy regimen is selected, effective treatment of CNS disease is an unmet need in BL. Novel targeted agents or immunotherapy that can overcome chemotherapy resistance, penetrate the blood-brain barrier, and are tolerated by adult patients of all ages offer the most promise. Sadly, the patients with the highest risk of treatment failure, such as those with CNS involvement or HIV, are often excluded from clinical trials testing these novel agents. ${ }^{8-10}$ Until we collectively change our minds about the wisdom of this practice, we should expect to be disappointed with 'real-world' outcomes.

\section{Disclosures \\ No conflicts of interest to disclose.}

\section{References}

1. Zayac AS, Evens A, Olszewski AJ. Outcomes of Burkitt lymphoma with central nervous system involvement: evidence from a large multi-center cohort study. Haematologica. 2021;106(7):1932-1942.

2. Mead GM, Sydes MR, Walewski J, et al. An international evaluation of CODOX-M and CODOX-M alternating with IVAC in adult Burkitt's lymphoma: results of United Kingdom Lymphoma Group LY06 study. Ann Oncol. 2002;13(8):1264-1274

3. Thomas DA, Faderl S, O'Brien S, et al. Chemoimmunotherapy with hyper-CVAD plus rituximab for the treatment of adult Burkitt and Burkitt-type lymphoma or acute lymphoblastic leukemia. Cancer. 2006;106(7):1569-1580.

4. Roschewski M, Dunleavy K, Abramson JS, et al. Multicenter study of risk-adapted therapy with dose-adjusted EPOCH-R in adults with untreated Burkitt lymphoma. J Clin Oncol. 2020;38(22):2519-2529.

5. Evens AM, Danilov AV, Jagadeesh D, et al. Burkitt lymphoma in the modern era: real world outcomes and prognostication across 30 US cancer centers. Blood. 2021;137(3):374-386.

6. Costa LJ, Xavier AC, Wahlquist AE, Hill EG. Trends in survival of patients with Burkitt lymphoma/leukemia in the USA: an analysis of 3691 cases. Blood. 2013;121(24):4861-4866.

7. Kelly JL, Toothaker SR, Ciminello L, et al. Outcomes of patients with Burkitt lymphoma older than age 40 treated with intensive chemotherapeutic regimens. Clin Lymphoma Myeloma. 2009;9(4):307-310

8. Uldrick TS, Ison G, Rudek MA, et al. Modernizing clinical trial eligibility criteria: recommendations of the American Society of Clinical Oncology-Friends of Cancer Research HIV Working Group. J Clin Oncol. 2017;35(33):3774-3780.

9. Lin NU, Prowell T, Tan AR, et al. Modernizing clinical trial eligibility criteria: recommendations of the American Society of Clinical Oncology-Friends of Cancer Research Brain Metastases Working Group. J Clin Oncol. 2017;35(33):3760-3773.

10. Lichtman SM, Harvey RD, Damiette Smit MA, et al. Modernizing clinical trial eligibility criteria: recommendations of the American Society of Clinical Oncology-Friends of Cancer Research Organ Dysfunction, Prior or Concurrent Malignancy, and Comorbidities Working Group. J Clin Oncol. 2017;35(33):3753-3759. 